\begin{tabular}{|l|l|l|l|l|}
\hline Cryst. Res. Technol. & $\mathbf{3 6}$ & 2001 & 12 & $1393-1398$ \\
\hline
\end{tabular}

\author{
N. M. GASANLY, A. AYDINLI* H. ÖZKAN \\ Department of Physics, Middle East Technical University, Ankara, Turkey \\ *Department of Physics, Bilkent University, Ankara, Turkey
}

\title{
Resonant Raman Scattering near the Free-to-Bound Transition in Undoped p-GaSe
}

\begin{abstract}
Raman spectra of GaSe layered crystal have been measured using a He-Ne laser and temperature tuning the free-to-bound gap in the range 10-290 K. Resonance enhancement of $E^{\prime \prime}{ }^{(2)}$ mode has been observed for both incident and scattered photon energies equal to the free-to-bound transition energy.
\end{abstract}

Keywords: resonant Raman scattering, semiconductor compounds, layered crystals, GaSe

(Received June 18, 2001; Accepted July 17, 2001)

\section{Introduction}

Resonant Raman scattering (RRS) around excitonic transitions in layered crystals has been a subject of extensive investigation in recent years (ASHOKAN et al.; BALTOG et al; JULIEN et al.; NAKAOKA et al.; SCAMARCIO et al.). Among various semiconductors, the layered compound GaSe seems to be particularly worth studying. In GaSe, the conduction band has an absolute minimum at $\mathbf{k} \neq 0$ (indirect gap) and a relative minimum at $\mathbf{k}=0$ (direct gap). As-grown $\varepsilon$-GaSe is an $p$-type semiconductor having an indirect and direct band gaps $E_{g i}=$ 2.103 and $E_{g d}=2.128 \mathrm{eV}$ at $4.2 \mathrm{~K}$. It has direct as well as indirect exciton states within 55 meV below the direct gap energy (VOITCHOVSKY, MERCIER).

Observation of one-phonon RRS in $\varepsilon$-GaSe crystal as the exciting laser frequency varies around direct and indirect free exciton transitions has already been reported by several authors (BALKANSKI et al; CAMASSEL et al.; CHIANG et al.; HDF, IRWIN; REYDELLET, BESSON). Because of strong Fröhlich interaction only the $A_{2}^{\prime(4)}(L O)$ and $E^{\prime(4)}(L O)$ modes at 247.0 and $253.2 \mathrm{~cm}^{-1}$, respectively, show strong resonant enhancement, whereas $A_{1}^{\prime(4)}(134.1$ $\left.\mathrm{cm}^{-1}\right)$ and $A_{1}^{\prime(2)}\left(307.8 \mathrm{~cm}^{-1}\right)$ modes show first an anti-resonant and then resonant behavior as the exciting laser energy approaches the direct free exciton energy (HOFF, IRWIN). Resonance in the Raman cross-section for $A_{1}^{\prime(4)}, A_{2}^{\prime \prime(4)}(L O)$ and $E^{\prime(4)}(L O)$ modes of GaSe at $80 \mathrm{~K}$ has been observed for both incident and scattered photon energies equal to the direct free exciton energy (REYDELLET, BESSON). Double resonance in Raman scattering on $E^{\prime(4)}(L O)$ mode of GaSe at $77 \mathrm{~K}$ has also been studied (CHIANG et al.). It was shown that the dispersion of RRS is dominated by resonances with the direct and indirect free exciton states.

Typically, resonant enhancement of phonon Raman scattering takes place when the incoming and/or outgoing photon energy is resonant with a singularity in the energy band structure of the solid. Resonance is most often encountered through free and bound excitons. 
To our knowledge there is only one paper in literature (HENNING, KESSENER), in which the authors reveal a resonant enhancement of phonon Raman scattering in GaAs when the energy of the incoming light is resonant with a nonexcitonic (donor-to-acceptor) transition.

In this paper we report an observation of RRS when the energy of the incoming and outgoing light is resonant with free-to-bound transition in layered $\varepsilon$-GaSe crystal.

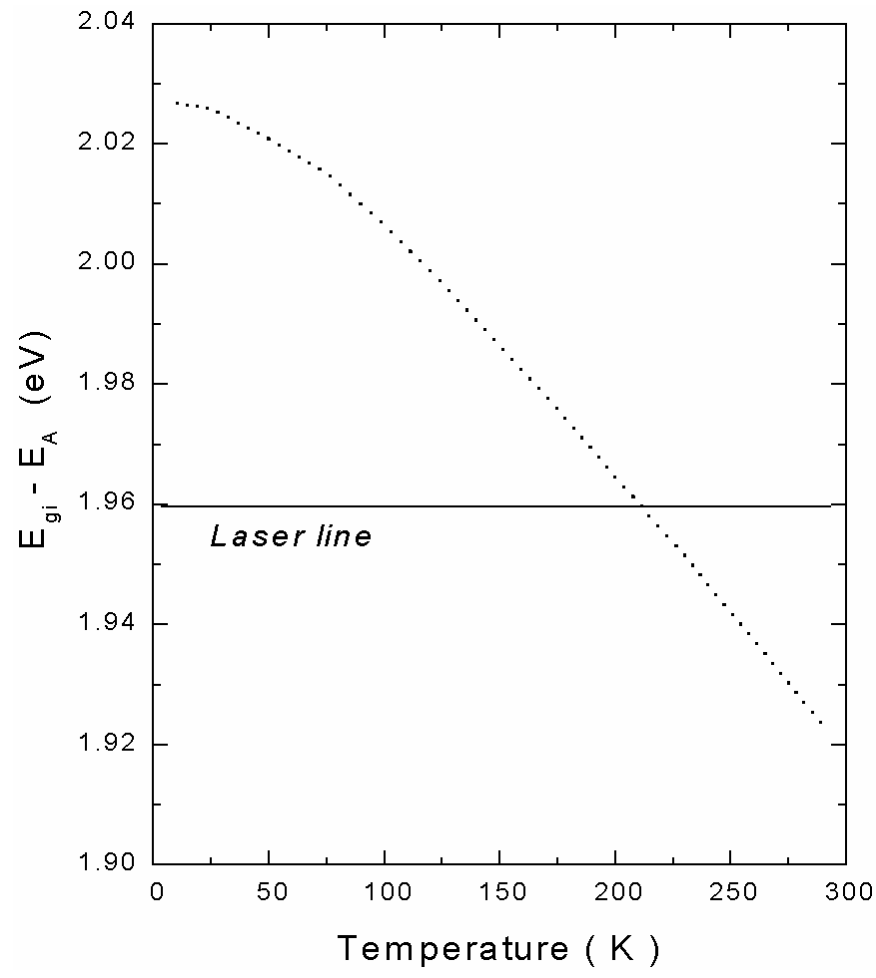

Fig. 1: Temperature dependence of free-to-bound transition energy.

\section{Experimental}

Gallium selenide polycrystals were synthesized from high-purity elements (at least 99.999\%) taken in stoichiometric proportions. $\varepsilon$-GaSe single crystals were grown by the modified Bridgman method. The analysis of X-ray diffraction data showed that they crystallize in hexagonal unit cell with parameters: $a=0.3745$ and $c=1.5921 \mathrm{~nm}$. Sample suitable for measurements was obtained by easy cleavage perpendicular to optical $c$-axis, which is parallel to $z$-axis of the crystal and perpendicular to the layer plane. Alongside with two natural cleavage planes, the sample had also two ground and polished surfaces perpendicular to them and forming a right angle between themselves. Such a treatment of the specimen made it possible to carry out the polarization measurements at any scattering geometries. Raman scattering measurements in GaSe layered crystal were performed in the right-angle scattering geometry in the frequency range 10 to $320 \mathrm{~cm}^{-1} .30 \mathrm{~mW} \mathrm{He}-\mathrm{Ne}$ laser $(632.8 \mathrm{~nm})$ was used as the exciting light source. The scattered light was analyzed using a U-1000 "Jobin Yvon" double grating spectrometer and a cooled GaAs photomultiplier supplied with necessary photon counting electronics. The Raman line positions were determined within an accuracy of $\pm 0.1 \mathrm{~cm}^{-1}$. A “ CTI-Cryogenics M-22" closed-cycle helium cryostat was used to 
cool the crystals from room temperature down to $10 \mathrm{~K}$. The temperature was controlled within an accuracy of $\pm 0.5 \mathrm{~K}$.

\section{Results and discussion}

In order to study the resonance, the Raman spectrum of $\varepsilon$-GaSe was recorded using a He-Ne laser and temperature tuning the gap. By varying the temperature between 10 and $290 \mathrm{~K}$, it was possible to tune the free-to-bound gap from 2.027 to $1.923 \mathrm{eV}$, through the He-Ne laser line $\left(h \mathrm{v}_{L}=1.959 \mathrm{eV}\right)$ (Fig. 1). The effect of changing the temperature is equivalent to changing the photon energy of the laser by an amount equal in magnitude and of opposite sign to the corresponding change in the free-to-bound gap. Thus, it was possible to measure the Raman spectrum for a fine mesh of scattering photon energies (or their temperature-tuned equivalent) covering the region from $\left(E_{g i}-E_{A}\right)-h v_{L}=+0.068$ to $\left(E_{g i}-E_{A}\right)-h v_{L}=-0.036$ $\mathrm{eV}$. In our previous study the presence of acceptor level located at $E_{A}=0.076 \mathrm{eV}$ above the top of the valence band in $p$-GaSe crystal was revealed by photoluminescence measurements in the temperature range of 10-290 K (AYDINLI et al.). This shallow acceptor level is associated with stacking faults, which is easy to produce in this crystals due to weak van der Waals forces between the layers.

Fig. 2: Resonant Raman spectra of GaSe at different temperatures.

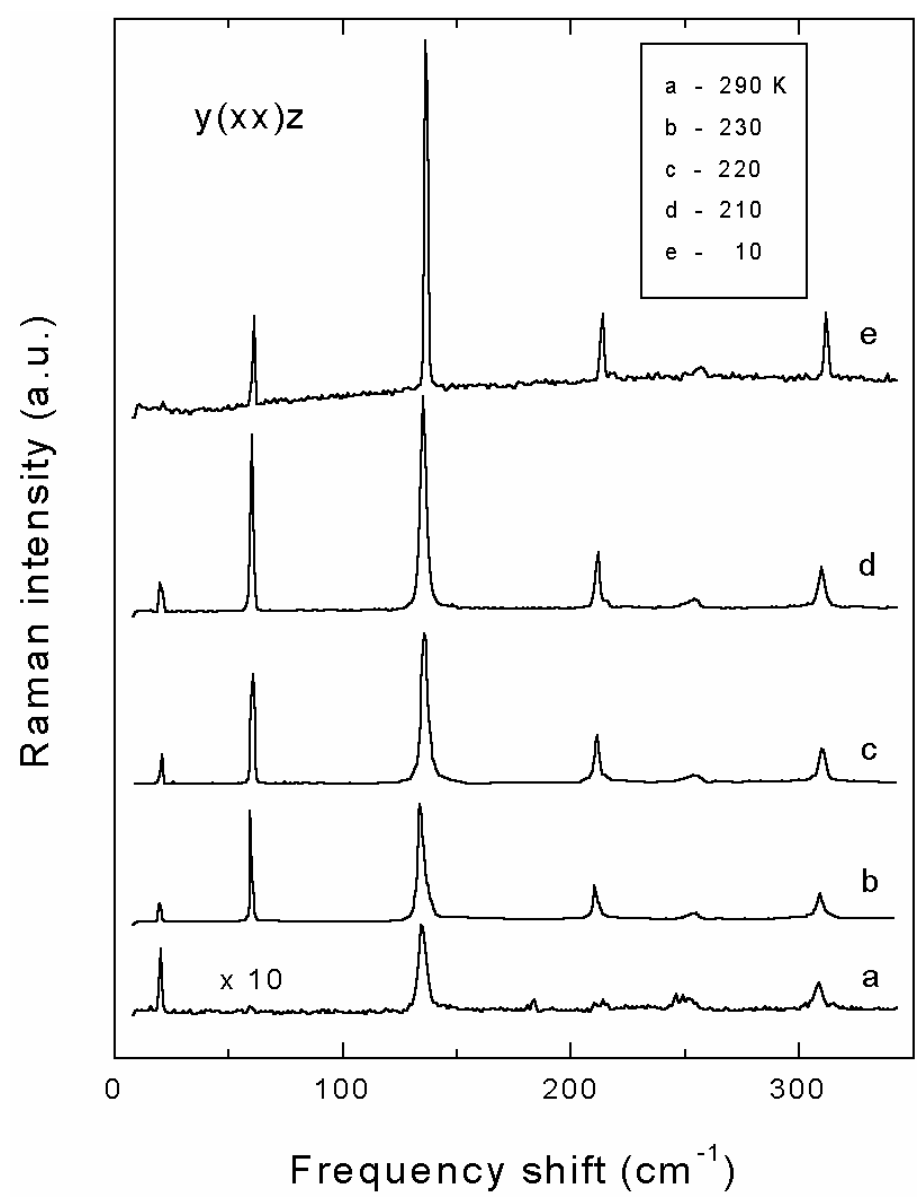


Figure 2 presents the Raman spectra of $\varepsilon$-GaSe crystal in $y(x x) z$ scattering geometry at different temperatures. A typical nonresonant Raman spectrum is shown in Fig. 2a. In $y(x x) z$ oriented spectra the compressional intralayer $A_{1}^{\prime(4)}\left(134.1 \mathrm{~cm}^{-1}\right)$ and $A_{1}^{\prime(2)}\left(307.8 \mathrm{~cm}^{-1}\right)$ modes and shear interlayer $E^{\prime(2)}\left(19.7 \mathrm{~cm}^{-1}\right)$ and intralayer $E^{\prime(4)}\left(213.5 \mathrm{~cm}^{-1}\right)$ modes are allowed. The shear nonpolar modes $E^{\prime(2)}\left(59.4 \mathrm{~cm}^{-1}\right)$ and $E^{\prime \prime(4)}\left(209.7 \mathrm{~cm}^{-1}\right)$ are Raman-active in the $(y z$ or $z x$ ) geometry. The scattering geometry $(x x)$ was chosen so that the normal off resonance $E^{\prime \prime}$ modes would be forbidden. It is known that the resonant enhancement of the modes in the allowed scattering configuration is weaker than those in the forbidden geometries. Furthermore, the enhancement is always strongest when both the incident and scattering radiations were polarized parallel to each other irrespective of the symmetry of the crystal (YU, CARDONA).

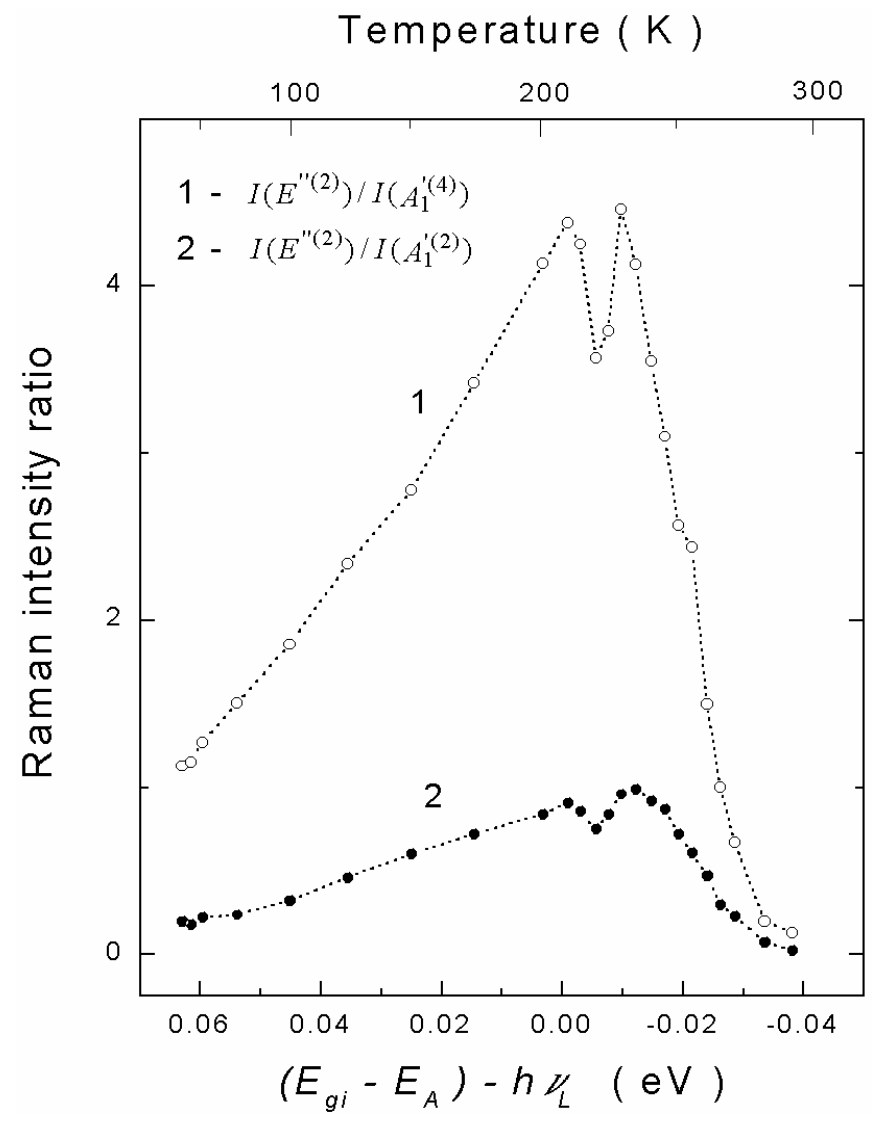

Fig. 3: Resonant behavior of the $E^{\prime \prime(2) ~ m o d e . ~ T h e ~ b r o k e n ~ c u r v e s ~}$ drawn through the data points to guide the eye.

The shear mode $E^{\prime(2)}\left(59.4 \mathrm{~cm}^{-1}\right)$ exhibit resonance maxima for incident and scattered photon energies equal to that of free-to-bound transition $\left(E_{g i}-E_{A}\right)$ (Fig. 2). The second $E^{\text {"(4) }}$ shear 
mode $\left(209.7 \mathrm{~cm}^{-1}\right)$ shows single resonance (not shown) at $210 \mathrm{~K}$ indicating that, this resonance is mediated through free-to-bound transitions as well. However, resonance of $E^{\prime \prime(4)}$ shear mode is much weaker and it is observed only with the incident light. In this report, we concentrate on the $E^{\prime \prime(2)}$ mode, which shows stronger resonance. The laser photon $h v_{L}$ excites the electron by means of indirect free-to-bound transition. Although momentum conservation forbids the direct excitation of indirect free-to-bound transition, stacking faults in the layered crystals can easily induce such a process (CHIANG et al.). The resonant enhancement of the mode arises through a vanishing denominator in the expression for the scattering cross section. The vanishing factor may be either $\left(E_{g i}-E_{A}\right)-h \mathrm{v}_{L}$ or $\left(E_{g i}-E_{A}\right)-$ $h \mathrm{v}_{S}$ (incoming or outgoing resonance (YU, CARDONA)). Here $\left(E_{g i}-E_{A}\right)$ is the resonant intermediate state energy. The difference between $h v_{L}$ and $h v_{S}$ is equal to the $E^{\prime \prime(2)}$ mode energy $(7.4 \mathrm{meV})$.

Figure 3 shows the resonant behavior of $E^{\prime(2)}$ mode of $\varepsilon$-GaSe in the region of the freeto-bound gap. With decreasing temperature down to $230 \mathrm{~K}$, Raman intensity ratios $I\left(E^{\prime \prime(2)}\right) / I\left(A_{1}^{\prime(4)}\right)$ and $I\left(E^{\prime(2)}\right) / I\left(A_{1}^{\prime(2)}\right)$ increase, then decrease reaching the minimum value at $220 \mathrm{~K}$ and again increase having a second maximum value at $210 \mathrm{~K}$. Below this temperature the intensity ratios decrease with a slower rate down to $10 \mathrm{~K}$.

The energy separation obtained between the position of two maxima is equal to $8.8 \mathrm{meV}$, which is in good agreement with the energy value of $7.4 \mathrm{meV}$ ( $E^{\prime \prime(2)}$ mode energy). This agreement supports our assignment of these two maxima to double resonance, which occurs due to the resonance of incident and scattered photons with free-to-bound transition in GaSe.

In conclusion, we have presented direct evidence of free-to-bound transition resonance in phonon Raman scattering of GaSe. Resonant enhancement of $E^{\text {"(2) }}$ mode was observed in GaSe when the energies of the exciting and scattered light coincide with free-to-bound transition energy. For the second shear mode, $E^{\prime(4)}$, we observe a weak single resonance.

\section{References}

Aydinli, A., Gasanly, N. M., Goksen, K.: Phil. Mag. Lett. 81 (2001) 859

Ashokan, S., Jain, K. P., Balkanski, M., Julien, C.: Phys. Rev. B 44 (1991) 11133

Balkanski, M., Reydellet, J., Hirliman, C., Kanehisa, M.: J. Luminescence 18/19 (1979) 665

Baltog, I., Lefrant, S., Minut, L., Mondescu, R.: Phys. Stat. Sol. (b) 176 (1993) 247

Camassel, J., Chiang, T. C., Shen, Y. R., Voitchovsky, J. P., Amer, N.M.: Solid State Commun. 19 (1976) 483

Chi ang, T. C., Camassel, J., Shen, Y. R., Voitchovsky, J. P.: Solid State Commun. 19 (1976) 157

Henning, J. C. M., Kessener, Y. A. R. R.: Semicond. Sci. Technol. 7 (1992) 1390

Hoff, R.M., Irwin, J.C.: Phys. Rev. B 10 (1974) 3464

Julien, C., Mavi, H. S., Jain, K. P., Balkanski, M., Perez-Vicente, C., Morales, J.: Mater. Sci. Eng. B 23 (1994) 98

Nakaoka, T., Wang, Y., Murase, K., Matsuda, O., Inoue, K.: Phys. Rev. B 61 (2000) 155692.

Reydellet, J., Besson, J. M.: Solid State Commun. 17 (1975) 23

Scamarcio, G., Cingolani, A., Lugara, M., Levy, F.: Phys. Rev. B 40 (1989) 1783 
Voitchovsky, J. P., Mercier, A.: Nuovo Cimento 22 (1974) 273

Yu, P., Cardona, M.: Fundamentals of Semiconductors, Springer, Berlin (1995), p. 362

\section{Contact information:}

Prof. Dr. N. M. GASANLY*,

Prof. Dr. H. OzKAN

Department of Physics, Middle East Technical Universty

06531 Ankara

Turkey

Prof. Dr. A. AYDINLI

Department of Physics

Bilkent University

06533 Ankara

Turkey

*corresponding author

e-mail: nizami@metu.edu.tr 